\title{
Stepwise Hydration of Ionized Aromatics. Energies, Structures of the Hydrated Benzene Cation, and the Mechanism of Deprotonation Reactions
}

\author{
Yehia M. Ibrahim, Edreese H. Alshraeh, Michael N. Meot-Ner (Mautner) and \\ M. Samy El-Shall* \\ Department of Chemistry, Virginia Commonwealth University, \\ Richmond, Virginia 23284
}

Steve Scheiner

Department of Chemistry, Utah State University, Logan, UT 84322

Supporting Information 


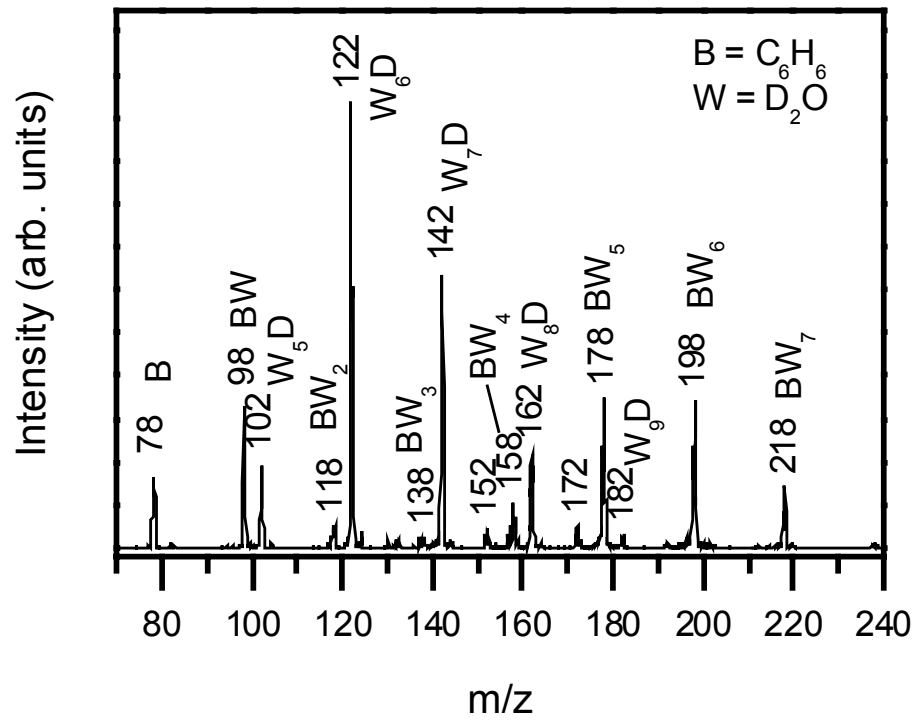

Figure S1: Mass spectrum obtained after injecting $\mathrm{C}_{6} \mathrm{H}_{6}{ }^{-+}$ions into 164 mtorr of neat $\mathrm{D}_{2} \mathrm{O}$ vapor at $245 \mathrm{~K}$ using $16 \mathrm{eV}$ (lab.) injection energy. In the $\mathrm{C}_{6} \mathrm{H}_{6}{ }^{-1}\left(\mathrm{D}_{2} \mathrm{O}\right)_{\mathrm{n}}$ series only the $\mathrm{m} / \mathrm{z} 78,98,118,138,158,178,198$ and 218 ions are observed but not the H/D exchanged ions $\mathrm{C}_{6} \mathrm{H}_{5}{ }^{\circ}\left(\mathrm{D}_{2} \mathrm{O}\right)_{\mathrm{n}} \mathrm{D}^{+}$ions one $\mathrm{m} / \mathrm{z}$ unit higher. 


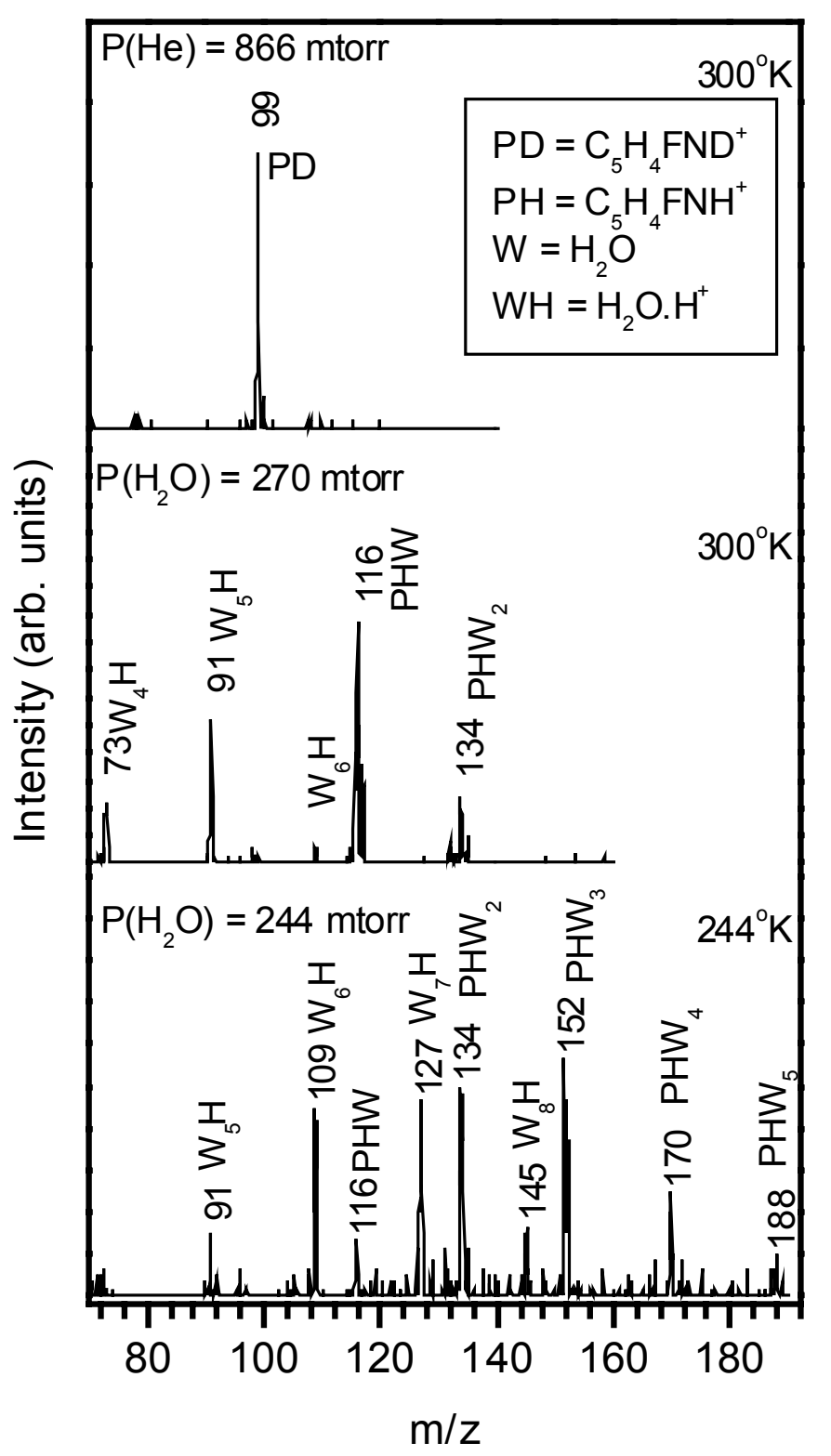

Figure S2: Mass spectrum obtained after injecting 2-FpyridineD ${ }^{+}\left(2-\mathrm{FC}_{5} \mathrm{H}_{4} \mathrm{ND}^{+}, \mathrm{m} / \mathrm{z}\right.$ 99) ions into $\mathrm{He}$ or into $\mathrm{H}_{2} \mathrm{O}$ vapor using $16 \mathrm{eV}$ (lab.) injection energy at the temperatures and pressures indicated. In the 2-Fpyridine-water cluster series $\left(\mathrm{PHW}_{\mathrm{n}}\right)$ only the 2$\mathrm{FC}_{5} \mathrm{H}_{4} \mathrm{NH}^{+}\left(\mathrm{H}_{2} \mathrm{O}\right)_{\mathrm{n}}$ ions, $\mathrm{m} / \mathrm{z} 116,134,152,170,188$ are observed where $\mathrm{D}^{+}$has exchanged for $\mathrm{H}^{+}$but not the unexchanged ions $2-\mathrm{FC}_{5} \mathrm{H}_{4} \mathrm{ND}^{+}\left(\mathrm{H}_{2} \mathrm{O}\right)_{n}$ one $\mathrm{m} / \mathrm{z}$ unit higher. 


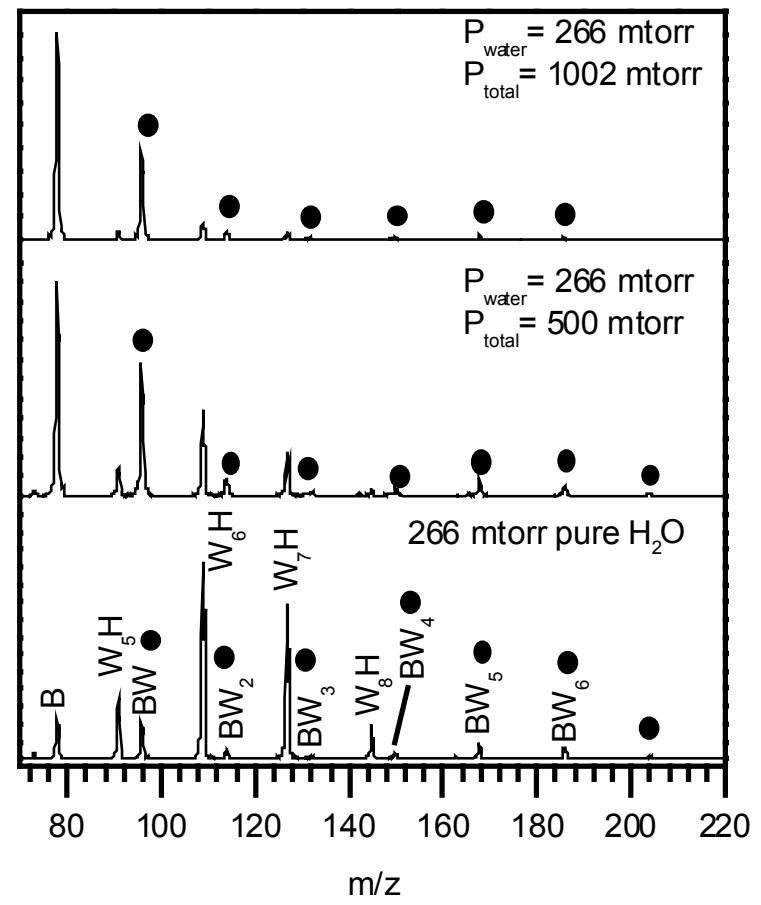

Figure S3. Effects of adding third-body He gas to the benzene/water system. Benzene ions are injected using $18 \mathrm{eV}, 30 \mathrm{~V}$ drift voltage and $253 \mathrm{~K}$. Increasing third-body $\mathrm{He}$ pressure quenches deprotonation to form the $\left(\mathrm{H}_{2} \mathrm{O}\right)_{n} \mathrm{H}^{+}$ions $\left(\mathrm{W}_{\mathrm{n}} \mathrm{H}\right)$. 
Complete Ref. 36.

Gaussian 03, Revision C.02, Frisch, M. J.; Trucks, G. W.; Schlegel, H. B.; Scuseria, G. E.; Robb, M. A.; Cheeseman, J. R.; Montgomery, J. A., Jr.; Vreven, T.; Kudin, K. N.; Burant, J. C.; Millam, J. M.; Iyengar, S. S.; Tomasi, J.; Barone, V.; Mennucci, B.; Cossi, M.; Scalmani, G.; Rega, N.; Petersson, G. A.; Nakatsuji, H.; Hada, M.; Ehara, M.; Toyota, K.; Fukuda, R.; Hasegawa, J.; Ishida, M.; Nakajima, T.; Honda, Y.; Kitao, O.; Nakai, H.; Klene, M.; Li, X.; Knox, J. E.; Hratchian, H. P.; Cross, J. B.; Bakken, V.; Adamo, C.; Jaramillo, J.; Gomperts, R.; Stratmann, R. E.; Yazyev, O.; Austin, A. J.; Cammi, R.; Pomelli, C.; Ochterski, J. W.; Ayala, P. Y.; Morokuma, K.; Voth, G. A.; Salvador, P.; Dannenberg, J. J.; Zakrzewski, V. G.; Dapprich, S.; Daniels, A. D.; Strain, M. C.; Farkas, O.; Malick, D. K.; Rabuck, A. D.; Raghavachari, K.; Foresman, J. B.; Ortiz, J. V.; Cui, Q.; Baboul, A. G.; Clifford, S.; Cioslowski, J.; Stefanov, B. B.; Liu, G.; Liashenko, A.; Piskorz, P.; Komaromi, I.; Martin, R. L.; Fox, D. J.; Keith, T.; Al-Laham, M. A.; Peng, C. Y.; Nanayakkara, A.; Challacombe, M.; Gill, P. M. W.; Johnson, B.; Chen, W.; Wong, M. W.; Gonzalez, C.; Pople, J. A., Gaussian, Inc, 2004. 